\title{
A CFD study on the mechanisms which cause cavitation in positive displacement reciprocating pumps
}

\author{
Aldo Iannetti ${ }^{1}$, Matthew T. Stickland ${ }^{1}$ and William M. Dempster ${ }^{1}$ \\ 1. Department of Mechanical and Aerospace engineering, University of Strathclyde, Glasgow G1 1XJ, UK
}

\begin{abstract}
A transient multiphase CFD model was set up to investigate the main causes which lead to cavitation in positive displacement (PD) reciprocating pumps. Many authors such as Karsten Opitz [1] agree on distinguishing two different types of cavitation affecting PD pumps: flow induced cavitation and cavitation due to expansion. The flow induced cavitation affects the zones of high fluid velocity and consequent low static pressure e.g. the valve-seat volume gap while the cavitation due to expansion can be detected in zones where the decompression effects are important e.g. in the vicinity of the plunger. This second factor is a distinctive feature of PD pumps since other devices such as centrifugal pumps are only affected by the flow induced type. Unlike what has been published in the technical literature to date, where analysis of positive displacement pumps are based exclusively on experimental or analytic methods, the work presented in this paper is based entirely on a CFD approach, it discusses the appearance and the dynamics of these two phenomena throughout an entire pumping cycle pointing out the potential of CFD techniques in studying the causes of cavitation and assessing the consequent loss of performance in positive displacement pumps.
\end{abstract}

Key words: Multiphase flows, PD reciprocating pump, cavitation model, expansion cavitation, flow induced cavitation

\section{Introduction}

The phenomenon of cavitation in pumps is still a complex problem to study. If one focuses on the sole category of positive displacement $(\mathrm{PD})$ reciprocating pumps one may say that there is a significant shortage of technical literature in this important area. Concentrating on the numerical analysis literature, very few CFD works on PD reciprocating pumps have been made so far, none of them deals with a comprehensive model of this kind of device operating in cavitation regimes. The main reason for the lack of studies dealing with the numerical analysis of cavitation dynamics in PD pumps is a consequence of the following reasons:

- Over the last decades PD pumps have gradually become obsolete compared to

Corresponding author: Aldo Iannetti, master, main research field: fluid dynamics. E-mail: aldo.iannetti@strath.ac.uk centrifugal pumps on which great effort has been spent by researchers both in experimental and numerical analysis. This was recalled by Herbert Tackett [2] who identifies the cause of the great popularity of centrifugal pumps due to the technological improvement made to them in the last decades. He also pointed out that, as a consequence, PD pumps nowadays may be considered a technically "old" device.

- Despite their appearance PD pumps are a complex device to model and study particularly by means of CFD. This has led the few researchers involved in PD pumps studies to prefer experimental tests over numerical methods.

The experimental methods, which are the only techniques utilized so far, usually provide the analysts with all the difficulties related to how to take, from the test rigs, crucial information such as 


\section{A CFD Study on the mechanisms which cause cavitation in positive displacement reciprocating pumps}

the pressure field, the production rate of water vapour and the loss of volumetric efficiency. Furthermore numerical methods have not been feasible for many years because of the great amount of computational resources that a complex model, such a pump in cavitating condition, may need. Herbert Tackett [2] also explains that there are still many applications where PD pumps outperform centrifugal pumps which is the reason why, in the authors' opinion, in the next few years a re-evaluation of this "old" device is to be expected. One of the reasons for the re-evaluation lies in the development of both High Performance Computational (HPC) systems and CFD techniques such as multiphase algorithms and moving meshes which provide the analysts with advanced numerical tools ready to be employed in the analysis of fluid dynamics in PD pumps despite their complexity, will be demonstrated in this paper.

The main feature successfully implemented in the model developed by the authors, which puts this work ahead of the previous work such as that carried out by Ragoth Singh [3], is the simultaneous coexistence of the following sub-models:

1. Compressibility of water. Even though water, in certain operative condition, may be considered incompressible there are periods within the pumping cycle when the inlet and outlet valve are both closed and the compressibility model is required to stabilize the simulation and fulfil the mass continuity equation.

2. The valve dynamics model. The inlet and outlet valves move following the pump chamber pressure field which in turn depends on the valves dynamics. To correctly model a PD pump it is crucial to provide the solver with a User Defined Function (UDF) which accounts for the two-way coupling between the valve dynamics and the pressure field. As stated by Stephen Price [4], cavitation strongly depends on the inertia characteristic of the valve.

3. Advanced cavitation model. The choice of the cavitation model is crucial to achieve reasonably accurate results in the case of full cavitation conditions because the analyst must account for the noncondensable gas mass fraction to predict pump performance deterioration in the cavitating conditions. As demonstrated by H. Ding [5] the amount of non-condensable gas dissolved in the water affects the prediction of the minimum Net Positive Suction Head (NPSH) required in the inlet manifold to keep the volumetric efficiency loss above the generally accepted $3 \%$ as recalled by John Miller [6].

The important role of the non-condensable gasses in cavitation was also pointed out by Tillmann Baur [7] who carried out an experimental test to demonstrate the interaction of the gases dissolved in the water on the bubble dynamics.

Many authors such as Karsten Opitz [8] agree on the partitioning of the cavitation types into incipient (also referred to as marginal cavitation), partial and full cavitation. They are characterized by different features as described in [8] and it is of crucial importance, for the designer, to know which cavitating condition the pump being designed will operate in. In the case of incipient or marginal cavitation, for instance, it is understood [1] that the number of bubbles and their distribution do not seem to be harmful to the pump and, avoiding any operating condition in this range, would result in a uneconomical device. In the case of partial to full cavitation the damage as well as the loss in performance may be extremely high and allowing the pump to operate at that condition would result in failures and loss of money. 


\section{A CFD Study on the mechanisms which cause cavitation in positive displacement reciprocating pumps}

The cavitation phenomenon in PD pumps appears to be different from the one occurring in centrifugal pumps. In the latter case cavitation is related to the low pressure induced by the high velocity which affects the rotor at certain operational conditions (flow induced cavitation) while, in the case of PD pumps, cavitation may depend on the low static pressure due to the plunger decompression at the beginning of the inlet stroke as well as on the high velocity that the flow through the inlet valve may

\section{Material and Methods}

The transient CFD model simulated the entire pumping cycle; the induction stroke, from the Tod Dead Centre position (TDC) to when the plunger reached the Bottom Dead Centre (BDC) position sweeping through the displacement volume, to the delivery stroke when the plunger again reached the TDC position as shown in

Figure 1. The overall pumping cycle was included within the range $0^{\circ}-360^{\circ}$ of the reciprocating crank rotation where $0^{\circ}$ (plunger at TDC position) was the initial time of the induction stroke and $360^{\circ}$ (plunger at TDC position again) was the end of the delivery stroke. The 3D CAD model of the pump is shown in Figure 2 and was cleaned up and prepared from the $\mathrm{CAD}$ files used for manufacture for the Boolean experience. This was discussed by Karsten Opitz [1]. The work presented in this paper was based on a transient CFD model of a PD reciprocating plunger pump to investigate the cavitation dynamics in incipient to full cavitating conditions and discusses the rate of production/destruction of vapour in the vicinity of the plunger, where the flow velocity is small, and in the volume between the inlet valve and its seat where the velocities are high and the Bernoulli's effect is important.

operations which extracted the fluid volumes from the solid volumes The operation was performed with both valves in the closed position and the plunger located in the TDC position (initial simulation configuration). The fluid volume was then decomposed into the pattern shown in Figure 3 to allow the layering moving mesh algorithm [9] to correctly act during the simulation. Figure 3 shows that the displacement volume was created by means of creation of cell layers during the inlet stroke and removal of cell layers during the outlet stroke in the direction of the plunger axis. The layers created on the top of the plunger surface increased the overall fluid volume during the pumping cycle up to the displacement volume amount.

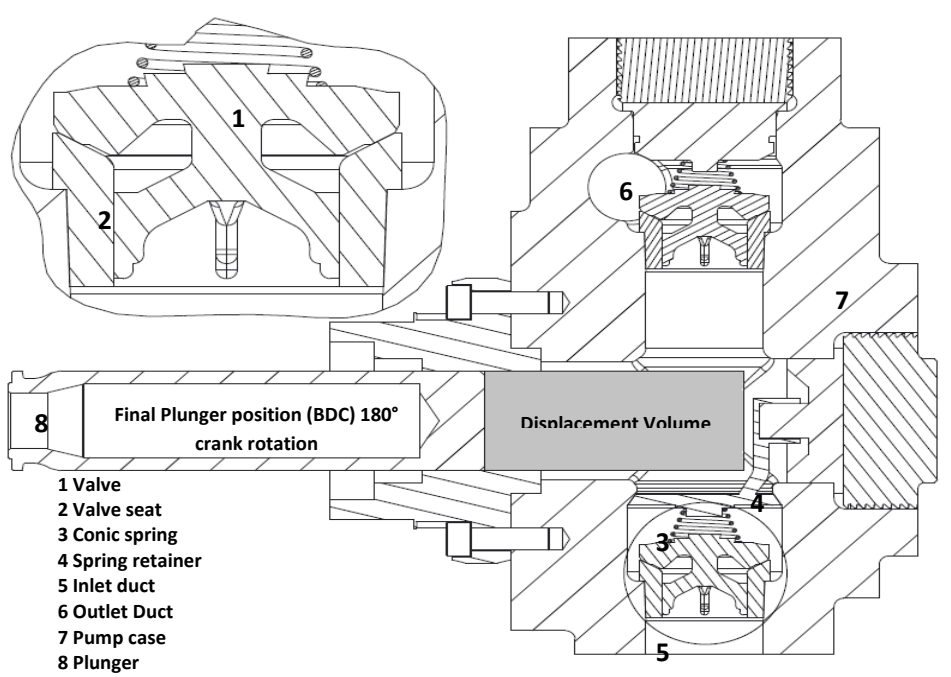

Figure 1. PD pump geometry and nomenclature. The displacement volume is swept by the plunger moving from TDC to BDC. 


\section{A CFD Study on the mechanisms which cause cavitation in positive displacement reciprocating pumps}

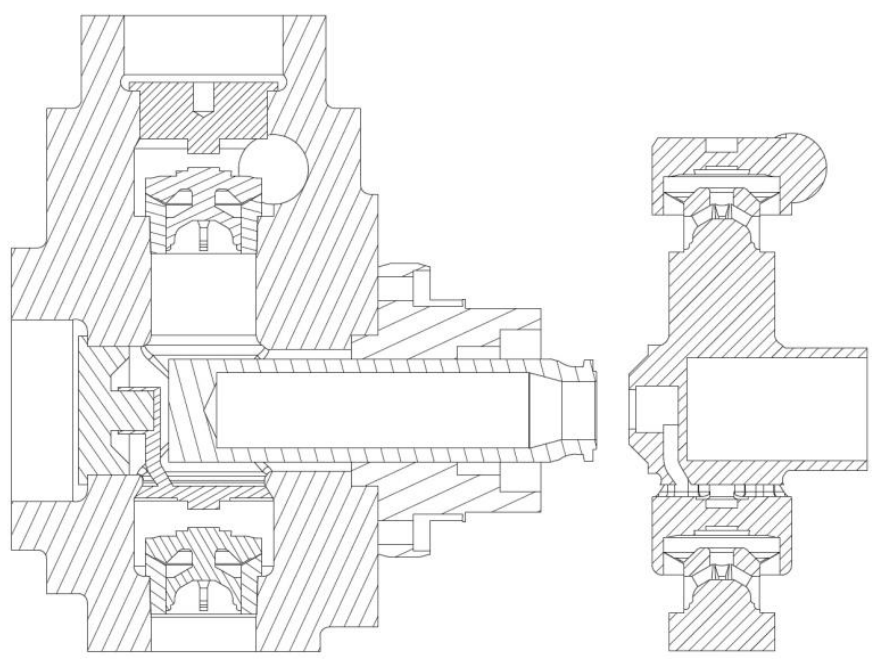

Figure 2. Generation of the fluid volumes from the 3D CAD model of the pump.
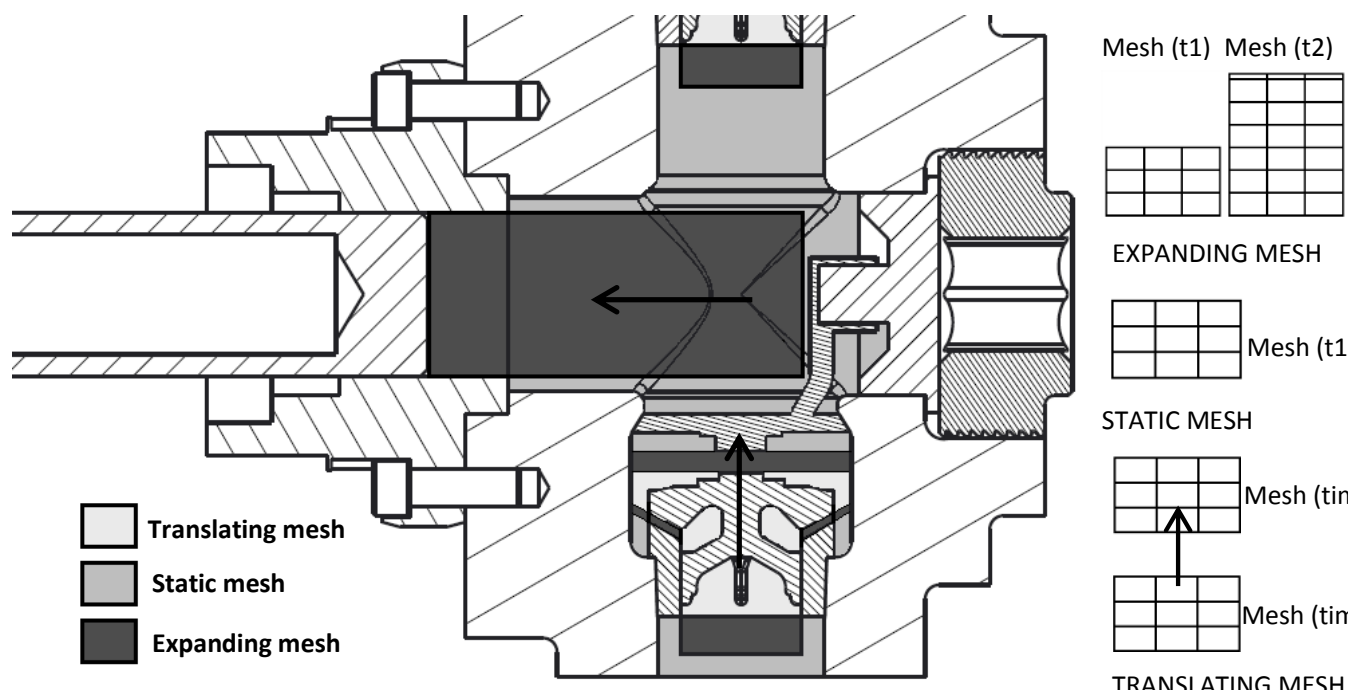

EXPANDING MESH

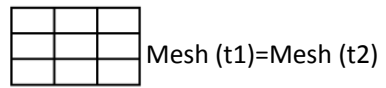

STATIC MESH

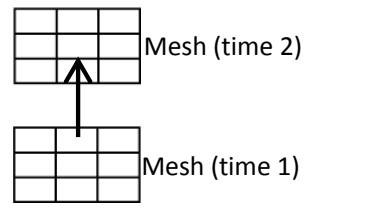

TRANSLATING MESH

Figure 3. Moving mesh: Decomposition pattern of fluid volumes, the arrows indicate the direction of creation of new mesh layers when the plunger is moving backwards (induction) and the valve is lifting up

The layers generation rate was a fixed time law which was automatically calculated by the solver by providing it with the reciprocating motion parameters (crank rotational speed and phase, connecting rod length and crank diameter). The solver utilised the In-Cylinder motion tool [9] to turn the set of reciprocating motion parameters into the plunger position (Figure 4) and speed and thus layer creation at each time step. To make this possible a full hexahedral mesh was chosen for the displacement volume. Figure 3 also shows how the valve lift was simulated. The fluid volume around the valve (inlet and outlet) was decomposed into either translating volumes or expanding volumes. During valve lift, the valve-seat gap volume was expanded by means of cell layer creation, the valve upper and lower volumes were rigidly translate upwards following the gap layering to keep the valve shape unchanged during the lift. The two cylindrical volumes on the top and on the bottom of the valve were compresed and expanded respectively to keep the volume continuity and to interface with the pump chamber static volumes, and vice versa while the valve closed. It is clear that during the valve motion, although the mesh changes, there was no increase in the overall fluid volume due to the motion of the valve. To make the valve lift possible a full hexahedral mesh was chosen for all the expanding and contracting volumes 


\section{A CFD Study on the mechanisms which cause cavitation in positive displacement reciprocating pumps}

as they were involved with the layering generation

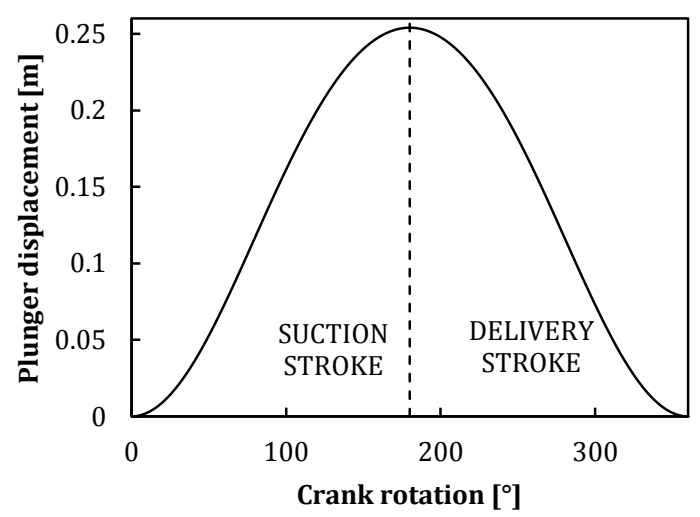

Figure 4. Boundary conditions, plunger displacement



Figure 5: Mass flow adjustable pressure drop for inlet and outlet boundary conditions.

All expanding volumes, were either cylindrical or annular shaped to simplify the meshing process and to permit a full hexahedral mesh. The static volumes and the translating volumes did not have any mesh requirements and a tetrahedral mesh was chosen for them.

Unlike the plunger, the valve layering generation was self-actuated. The diagram of Figure 6 summarises how the UDF managed to calculate the amount of valve lift to apply without any analyst's external action. The function at every time step utilised the pressure field output of the RANS solver to calculate the overall pressure force on the valve surfaces which was added to the spring force and then integrated to just like the plunger top surface. assess the valve velocity and displacement which was utilised by the moving mesh algorithm to update the valve position for the following time step. The spring force was provided to the UDF by means of spring stiffness characteristic curve. The function utilised the position of the valve at the previous time step to calculate the spring force to be applied to the valve force balance for the actual time step.

As mentioned in the introduction, the model was also equipped with a water compressibility model which was crucial to fulfil the mass continuity equation at the times when the inlet and outlet valve were both closed. The model made the assumption of one way coupling between the pressure field and the density field. This means that the pressure field affected the density field but the density did not affect the pressure. In this case the density field can be calculated implicitly without linking the pressure and density via the energy equation. The assumption is reasonable when the working fluid is water.

The distinguishing feature and added sub-model which improved the model presented in this document from the one discussed in [10] is the multiphase and cavitation algorithm. A three phase model composed of water, water vapour and $15 \mathrm{ppm}$ of non-condensable ideal gas was utilised as the working fluid. The water vapour fraction was initialised as null in all of the volumes and the Singhal et al. cavitation model managed the phase change dynamics according to the pressure field as explained in [11]. This cavitation model, also referred to as the "full" cavitation model, utilises a simple source term coming from the Rayleigh equation [12] by omitting the second-order derivative. It also accounts for the non-condensable gas effects already mentioned. A mass flow adjustable pressure was chosen as the boundary condition for the inlet and outlet pipe. Figure 5 shows that the solver automatically chose the static pressure 


\section{A CFD Study on the mechanisms which cause cavitation in positive displacement reciprocating pumps}

to apply according to the mass flow rate calculated

during the previous time step.

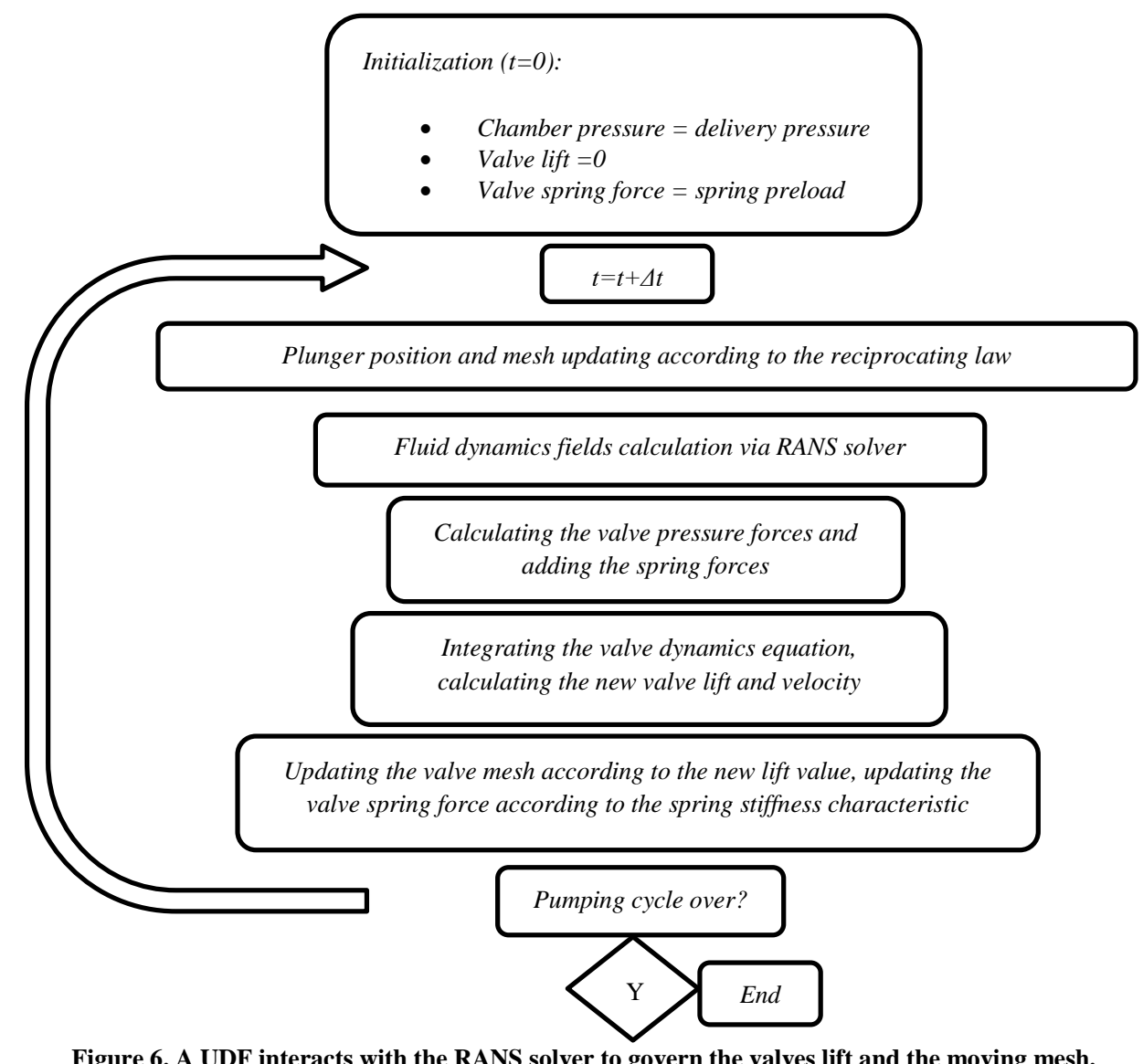

\subsection{Set up cases}

A mesh sensitivity analysis was carried out to define the best mesh size and spacing within the opposing needs of achieving good accuracy and keeping the computational time low. To this purpose three mesh sizes were tested; 3, 5 and 6 Million cells overall. The $5 \mathrm{M}$ cells model was chosen because it proved the best results at a lower computational time than the 6 million cells case.

The ANSYS-Fluent commercial code was chosen to solve the Reynolds Averaged Navier Stokes (RANS) equations and Table 1 shows a summary of the settings selected. The UDF was written in $\mathrm{C}++$ language. The standard $\mathrm{k}-\varepsilon$ model was chosen because it provided better convergence behaviour over other turbulence models such as the $\mathrm{k}-\omega$ but the "enhanced wall treatment" [13] was needed to deal with the problem of the low $\mathrm{y}^{+}$. In fact, at times during the pumping motion, the minimum flow velocity was zero as the reciprocating motion of the plunger started from the TDC position, this necessitaded the use of the "Enhanced wall treatment" which is capable of adjusting the standard wall functions in cases when (and where) the applicability of the standard wall function is no longer reasonable $\left(\mathrm{y}^{+}<100\right)$. A 12 GB RAM computer with an Intel Xeon W3670 @ $3.2 \mathrm{GHz}$ processor was employed for the simulation and the time needed for a single run (1 pumping cycle only) ranged from 3 to 4 weeks. A set of four cases dealing with four different boundary conditions in term of pressure inlet/outlet was launched. All cases assumed the flow to be isothermal and therefore the equation 


\section{A CFD Study on the mechanisms which cause cavitation in positive displacement reciprocating pumps}

of energy conservation was neglected.

Table 1. Solver settings

\begin{tabular}{|c|c|c|c|}
\hline Solver & & \multicolumn{2}{|c|}{ RANS, pressure based, transient } \\
\hline \multirow{5}{*}{ Models } & \multirow{3}{*}{ Multiphase } & \multicolumn{2}{|l|}{ Mixture model [13] } \\
\hline & & \multirow{2}{*}{ Phases } & $\begin{array}{l}\text { Water } \\
\text { liquid }\end{array}$ \\
\hline & & & Secondary phase \\
\hline & Turbulence & K- $\varepsilon$ Standard & Enhanced wall treatment \\
\hline & Cavitation & Singhal et al. & 15 ppm air (ideal gas) \\
\hline \multicolumn{2}{|c|}{ Pressure-Velocity coupling } & \multicolumn{2}{|l|}{ SIMPLE } \\
\hline \multirow{4}{*}{\multicolumn{2}{|c|}{ Spatial discretization }} & Momentum & Second order upwind \\
\hline & & Vapour & First order upwind \\
\hline & & Turbulent kinetic energy & Second order upwind \\
\hline & & Turbulent dissipation rate & Second order upwind \\
\hline \multicolumn{2}{|c|}{ Transient formulation } & \multicolumn{2}{|l|}{ First order implicit } \\
\hline \multirow{5}{*}{\multicolumn{2}{|c|}{ Under relaxation factors }} & Pressure & 0.3 \\
\hline & & Momentum & 0.7 \\
\hline & & Vapour & 0.5 \\
\hline & & Turbulent kinetic energy & 0.8 \\
\hline & & Turbulent dissipation rate & 0.8 \\
\hline \multicolumn{2}{|c|}{ Residuals } & \multicolumn{2}{|l|}{$10^{-3}$} \\
\hline \multicolumn{2}{|c|}{ Time step } & \multicolumn{2}{|c|}{$0.125^{\circ}$ crank rotation $\equiv 1.6 \times 10^{-4} \mathrm{~s} @ 130 \mathrm{rpm}$} \\
\hline \multicolumn{2}{|c|}{ Max Iteration per time step } & \multicolumn{2}{|l|}{35} \\
\hline \multirow{2}{*}{\multicolumn{2}{|c|}{ UDFs }} & \multicolumn{2}{|c|}{ Compressibility of water [10] } \\
\hline & & \multicolumn{2}{|l|}{ Valve dynamics, see figure 2} \\
\hline
\end{tabular}

Table 2. Boundary conditions summary

\begin{tabular}{llll}
\hline \multirow{2}{*}{ Case } & \multicolumn{2}{l}{ Pressure: function of the mass flow rate } & Plunger Displacement \\
\cline { 2 - 4 } & Inlet pressure $[\mathrm{kPaG}]$ & Outlet pressure $[\mathrm{kPaG}]$ & See figure 4 \\
\hline Case 1 & $0-\Delta \mathrm{P}($ see Figure 5$)$ & $0+\Delta \mathrm{P}($ see Figure 5$)$ & See figure 4 \\
\hline Case 2 & $25-\Delta \mathrm{P}($ see Figure 5$)$ & $25+\Delta \mathrm{P}($ see Figure 5$)$ & See figure 4 \\
\hline Case 3 & $50-\Delta \mathrm{P}($ see Figure 5$)$ & $50+\Delta \mathrm{P}($ see Figure 5$)$ & See figure 4 \\
\hline Case 4 & $100-\Delta \mathrm{P}($ see Figure 5$)$ & $100+\Delta \mathrm{P}($ see Figure 5$)$ & \\
\hline
\end{tabular}

The amount of non-condensable gas considered in the flow and listed in Table 1 behaved as ideal gas and its temperature variation under the overall pressure field was neglected. For all cases the inlet and outlet pressure were set as the sum of a constant value, ranging from $0 \mathrm{kPa}$ to $100 \mathrm{kPa}$ (depending on the case) and a transient value depending on the mass flow which was automatically calculated every time step by the solver. This added transient term took into account the distributed and concentrated pressure loss of the inlet and outlet pipelines. The reason for this added pressure drop term lies in the fact that the pressure loss is usually a characteristic curve of the pipe geometry which was not modelled. Because it was not under investigation, the authors decided to fix the curve to the one shown in Figure 5 which was fed into the solver by means of a piecewise linear law. Table 2 summarizes the four pressure boundary conditions for the four cases studied. A displacementtime law was chosen to drive the plunger and the moving mesh attached to it as previously explained, in a manner similar to [10]. The displacement history over crank rotation is shown in Figure 4. 


\section{A CFD Study on the mechanisms which cause cavitation in positive displacement reciprocating pumps}

\section{Results and Discussion}

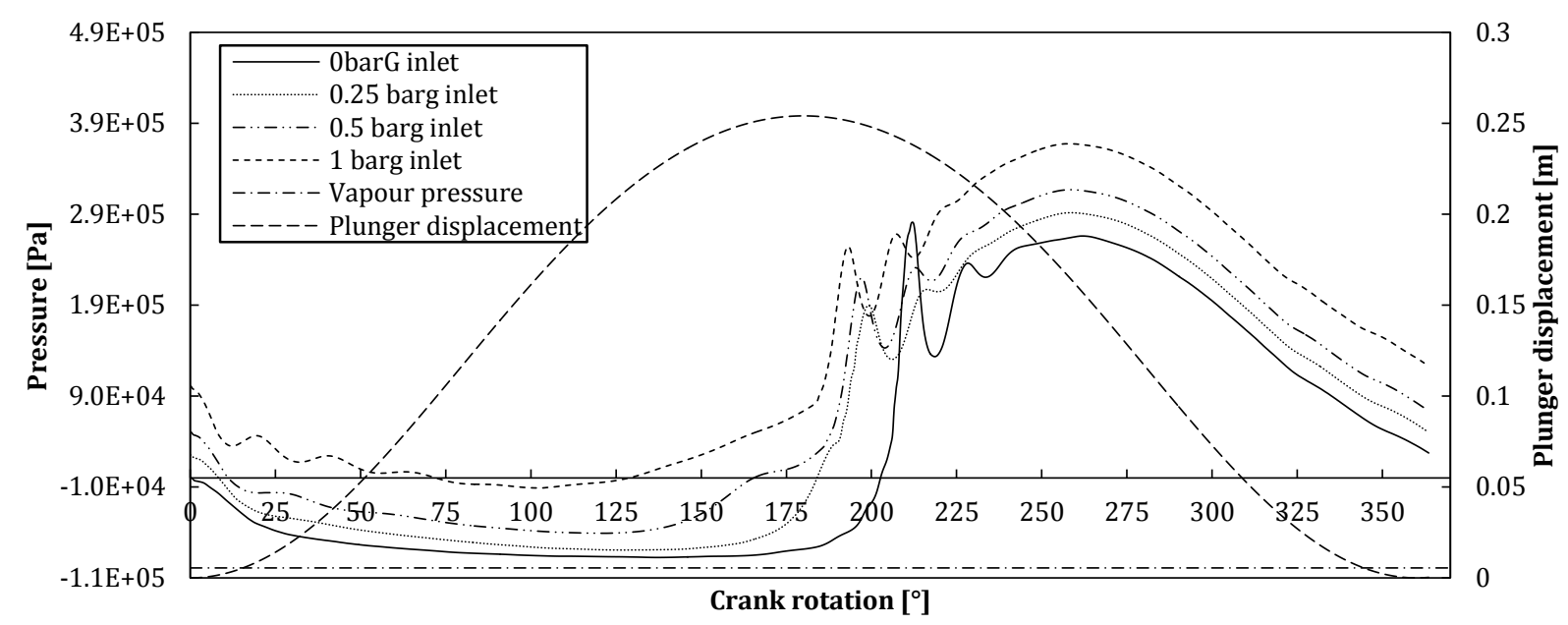

Figure 7. Chamber pressure-time histories, case 1 to 4 . The pressure is monitored in a fixed point near the TDC plunger position

\subsection{Case 1}

(Figure 7) The chamber pressure fell close to the vapour level and remained fairly constant throughout the temporal range $100^{\circ}-170^{\circ}$ of the inlet stroke. A significant amount of vapour was produced as shown in Figure 8 and in Figure 16. During the $17^{\circ}-70^{\circ}$ range the vapour volume fraction-time trend was steep demonstrating a high level of phase change, whereas it remained constant around $20 \%$ in the interval $70^{\circ}-190^{\circ}$. When the inlet stroke ceased $\left(180^{\circ}\right)$ the gap mean vapour volume fraction was still
$17.5 \%$, it quickly turned back to liquid water during the initial part of the outlet stroke $\left(180^{\circ}-200^{\circ}\right)$. Figure 9 shows that the phase change occurred also in the vicinity of the plunger and started very soon after the beginning of the induction stroke. Its trend may be considered linear ascending in the range $17^{\circ}-105^{\circ}$. Then a peak of $10 \%$ occurred and eventually showed a descending trend which terminated at $200^{\circ}$ of crank rotation.

Figure 8 and Figure 9 demonstrate the presence of two types of cavitation which occurred

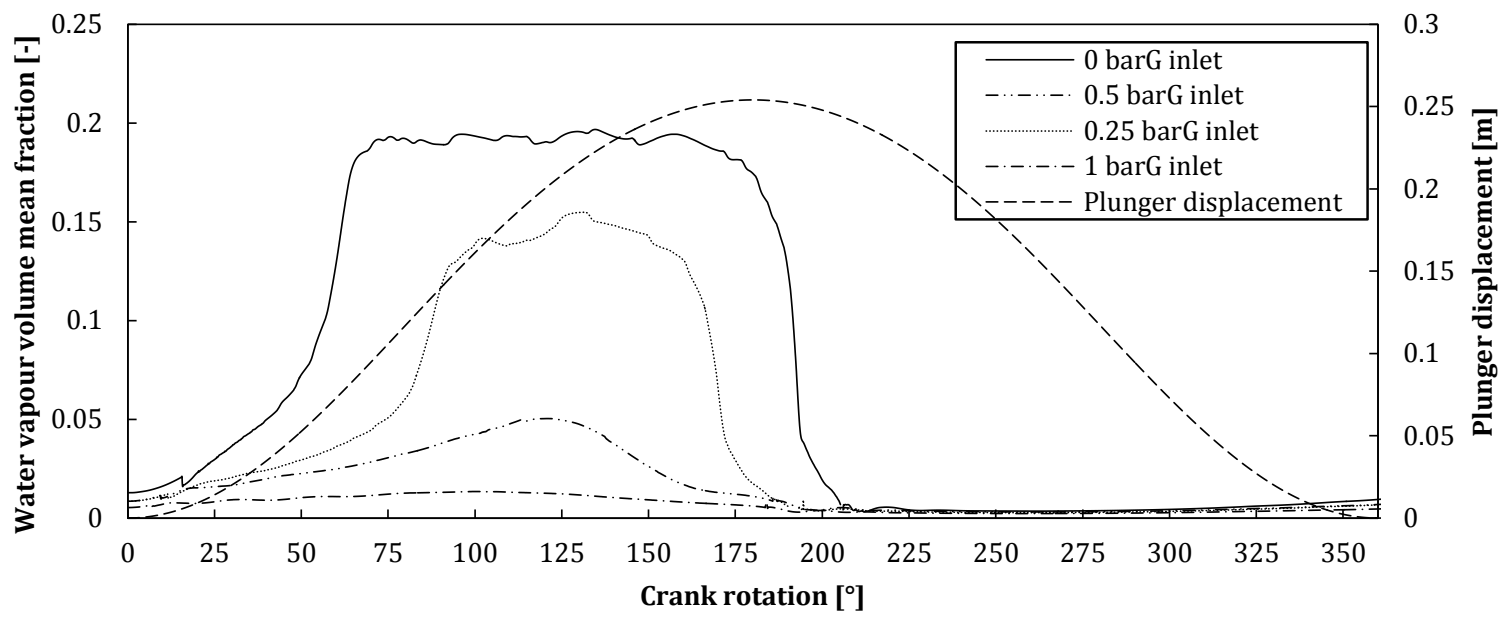

Figure 8. Inlet valve-seat gap volume mean vapour fraction, case 1 to 4 . 


\section{A CFD Study on the mechanisms which cause cavitation in positive displacement reciprocating pumps}

and low velocity regions such as the zones in the vicinity of the plunger where the maximum flow velocity can be considered equal to the plunger velocity (0 to $1.74 \mathrm{~m} / \mathrm{s})$ and insufficient to produce any flow induced cavitation effects. The second type, the flow induced cavitation, affected mainly the zones experiencing a high velocity flow such as the inlet valve-seat volume once the valve lifted up. Figure 8 , which quantifies the amount of vapour volume fraction present in the inlet valve-seat gap volume, shows a non-linear trend and a higher rate with respect to Figure 9. The rate of vapour fraction creation increased as the gap volume mean velocity and dynamic pressure increased (

Figure 10 and Figure 11) and caused the pressure drop (Bernoulli's effect). The delay in vapour condensation affected the inlet mass flow and the inlet valve lift histories as shown in Figure 12(a) and Figure 13(a). This effect is also shown in 


\section{A CFD Study on the mechanisms which cause cavitation in positive displacement reciprocating pumps}

Table 3 which quantifies it as $205.3^{\circ}, 25.3^{\circ}$ in the early stage of the outlet stroke. Figure 13(b) shows the consequent delay in outlet valve opening which Table 3 which quantifies it as $205.3^{\circ}, 25.3^{\circ}$ in the early stage of the outlet stroke. Figure 13(b) shows the consequent delay in outlet valve opening which

Table 3. According to the described phenomena, one can assume that the pump was operating at full cavitating conditions in accordance with Karsten Opitz [1], [8]. Figure 14 to Figure 16 present the contour plots of pressure, velocity and vapour was the reason for the $7 \%$ loss of volumetric efficiency shown

in was the reason for the $7 \%$ loss of volumetric efficiency shown

volume fraction respectively taken when the plunger reached $120^{\circ}$ of rotation (just after the peak of vapour generation) and qualitatively confirm the numerical trend of Figure 7 to Figure 13.

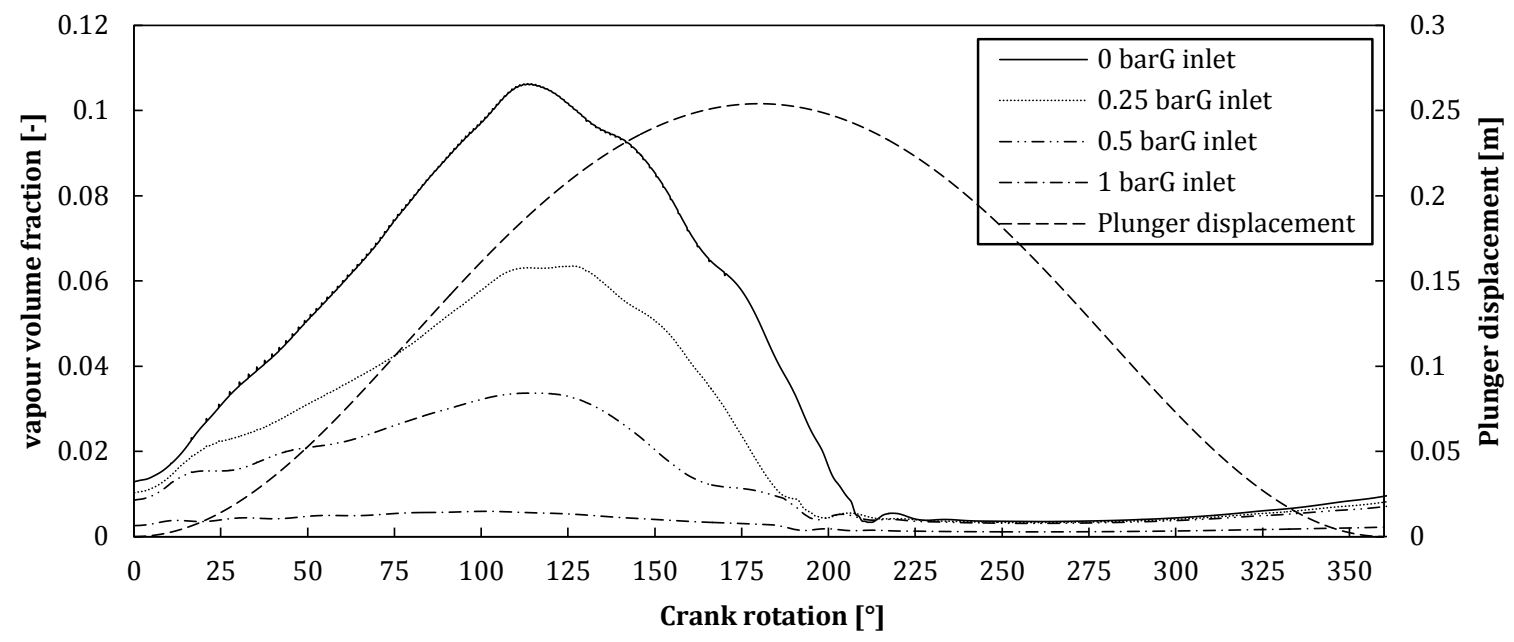

Figure 9. Plunger surfaces mean vapour fraction, case 1 to 4 .

\subsection{Case 2}

(Figure 7) The chamber monitor point pressure during the induction stroke approached the saturation vapour pressure. Figure 8 shows a behaviour of the vapour fraction similar to case 1 but the maximum values estimated by the CFD solver were lower $(15 \%)$ and remained almost constant over a narrower range $\left(90^{\circ}-165^{\circ}\right)$. It can be observed from Figure 9 that also, in the vicinity of the plunger, the vapour

Table 3 quantifies the delay of $14.6^{\circ}$ and a volumetric efficiency loss within the limit of $3 \%$ discussed by John Miller [6]. One can assume that case 2 describes a pump operating in the partial cavitating condition in accordance with Karsten Opitz [1], [8]. fraction follows a similar trend to case 1 with a smaller peak $(6 \%)$ and a linear increase in the vapour volume fraction but at a lower rate. All the observations on the flow induced cavitation and cavitation due to expansion made for case 1 are qualitatively valid also for case 2 . The smaller overall amount of vapour generated implied a smaller delay in valve closing which can be observed in Figure 13(a). 


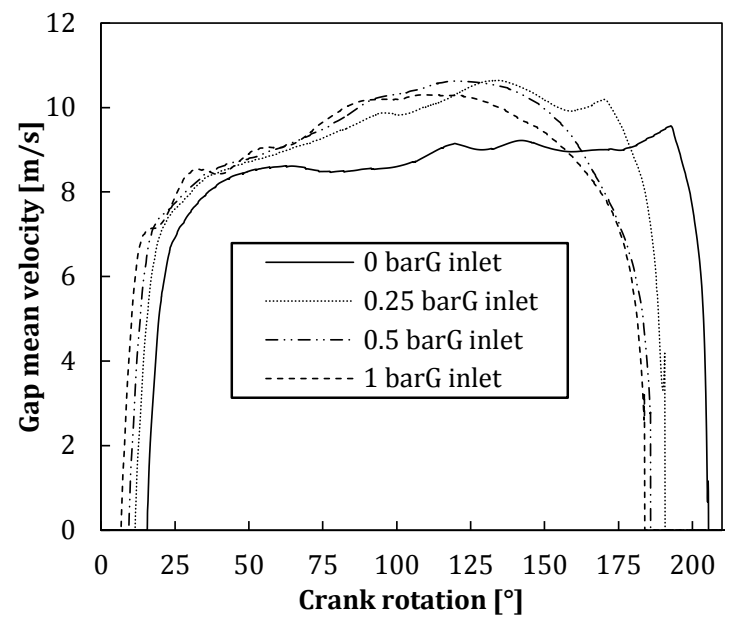

Figure 10. Inlet valve-seat gap volume mean flow velocity.

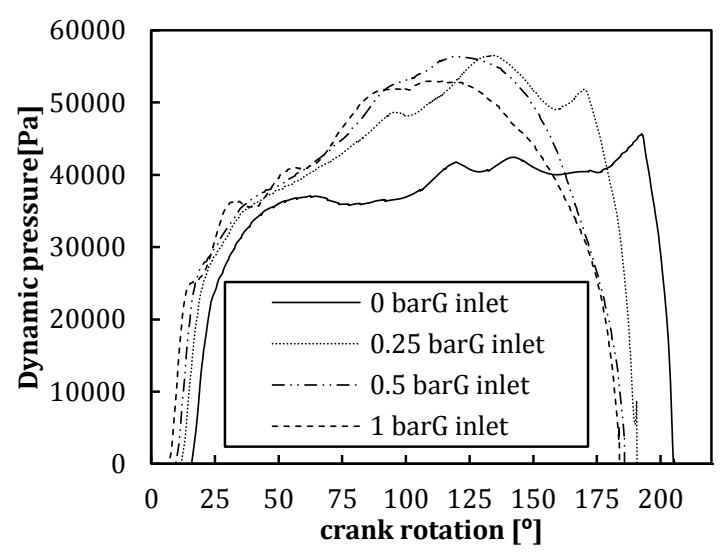

Figure 11. Inlet valve-seat gap volume mean dynamic pressure.

\subsection{Case 3}

Although the monitor pressure point in the chamber during the inlet stroke generally remained above the

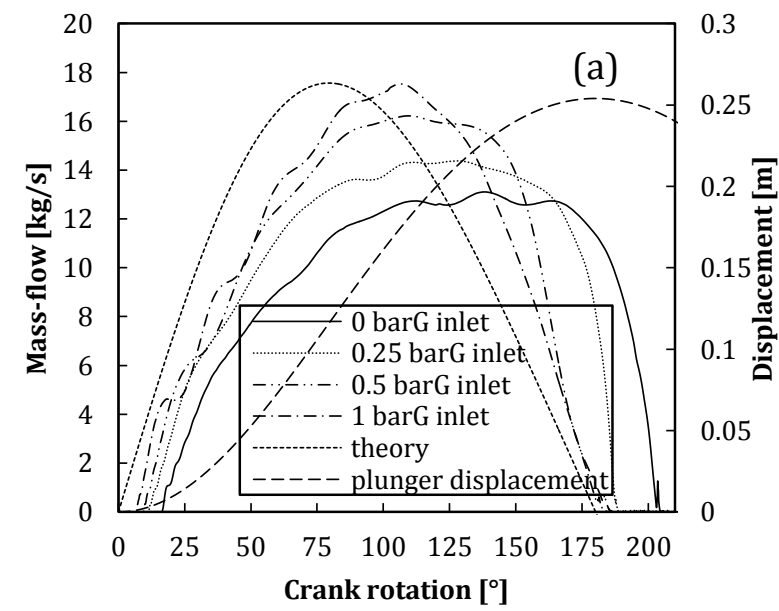

saturation vapour pressure (see Figure 7), a 5\% peak of vapour fraction was present in the gap volume as shown in Figure 8 and occurs at $120^{\circ}$ of crank rotation. One may say that on the whole the pressure remained above the vapour limit but locally there were regions affected by low pressure. In this case Figure 8 shows a linear trend which was different with respect to case 1 and 2 where the vapour volume fractions revealed a strongly non-linear behaviour before reaching the maximum.

In fact

Figure 10 points out that the high velocity in the inlet valve-seat gap volume, as well as the induced localized pressure drop, is a piece of evidence of the flow induced cavitation. The low level of vapour volume fraction resulted in a shorter delay of valve closing and an inlet mass flow rate/time history curve closer to the theoretical one (Figure 13 and Figure 12). The low level of volumetric efficiency loss $(<3 \%)$ implied that the pump was operating at the incipient cavitating condition since cavities do not disturb and modify the main flow as stated by JeanPierre Franc [14].

Figure 12. Inlet (a) and outlet (b) mass flow-time histories, case 1 to 4 . The theory curve is calculated as the positive displacement volume times the density of water at standard condition. 


\section{A CFD Study on the mechanisms which cause cavitation in positive displacement reciprocating pumps}
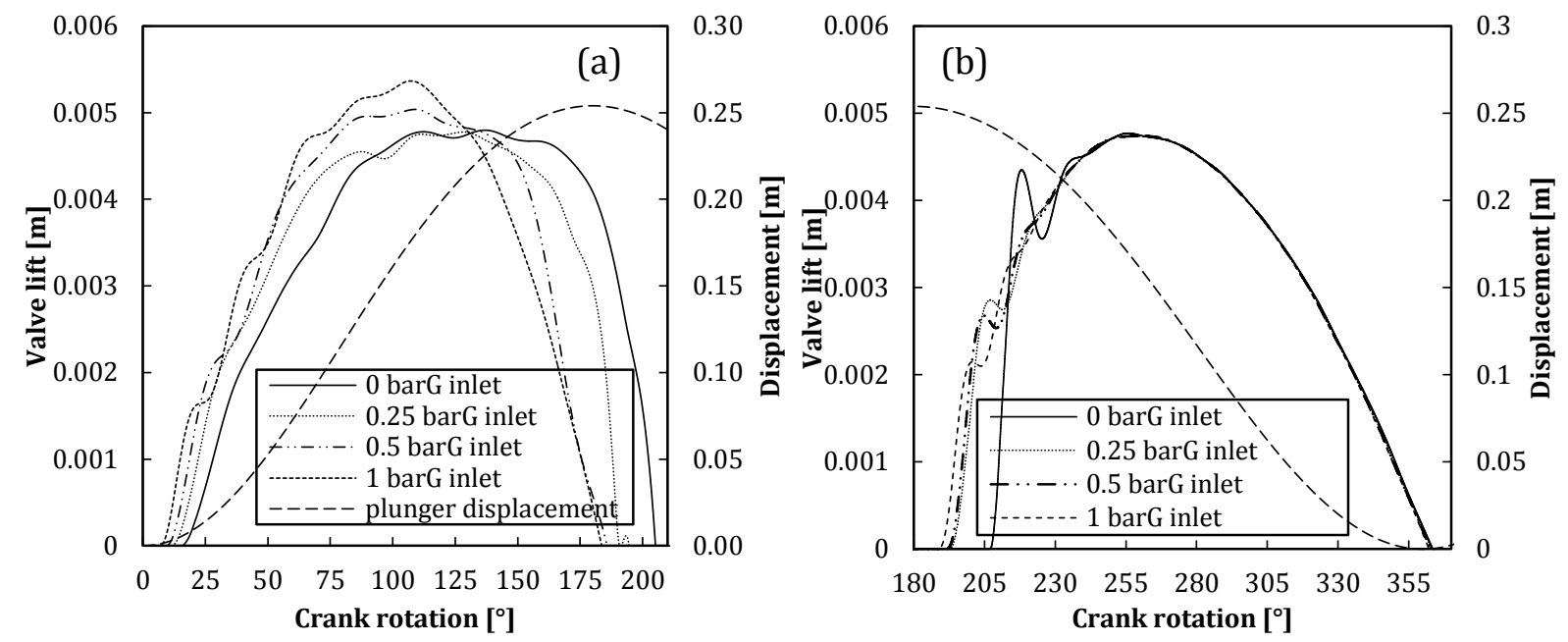

Figure 13. Inlet (a) and outlet (b) valve lift-time histories. Case 1 to 4.

\subsection{Case 4}

The chamber minimum pressure remained either generally or locally safely above the vapour limit, the minimum monitor point pressure/time curve ranged around the ambient pressure as shown in Figure 7. Figure 8 shows a flat trend of the vapour volume fraction throughout the pumping cycle. The graph indicates a $1 \%$ quantity of the second phase but, rather than water vapour this may be interpreted as the initial non-condensable mass fraction which slightly expanded during the inlet stroke. The model correctly calculated the expansion of that gas providing a minimum variation of its volume fraction. Such a phenomenon is commonly known as "gas cavitation" [7]. Among all cases this one is the closest to the theory in terms of inlet mass flow as pointed out by Figure 12 and it is affected by the least amount of volumetric efficiency loss (

Table 3).

Table 3.Volumetric efficiencies calculated relative to the standard condition (plunger stroke volume times water density at ambient pressure)

\begin{tabular}{lcccc}
\hline & $\begin{array}{c}\text { Inlet Max } \\
\text { pressure [kPa] }\end{array}$ & $\begin{array}{c}\text { Volumetric efficiency Vs } \\
\text { standard conditions [\%] }\end{array}$ & Inlet valve opening time $\left[{ }^{\circ}\right]$ & Inlet valve closing time $\left[^{\circ}\right]$ \\
\hline Case 1 & 0 & 93.36 & 15.6 & 205.3 \\
\hline Case 2 & 25 & 97.93 & 11.5 & 194.6 \\
\hline Case 3 & 50 & 98.26 & 9.6 & 185.8 \\
\hline Case 4 & 100 & 98.87 & 6.7 & 183.7 \\
\hline
\end{tabular}




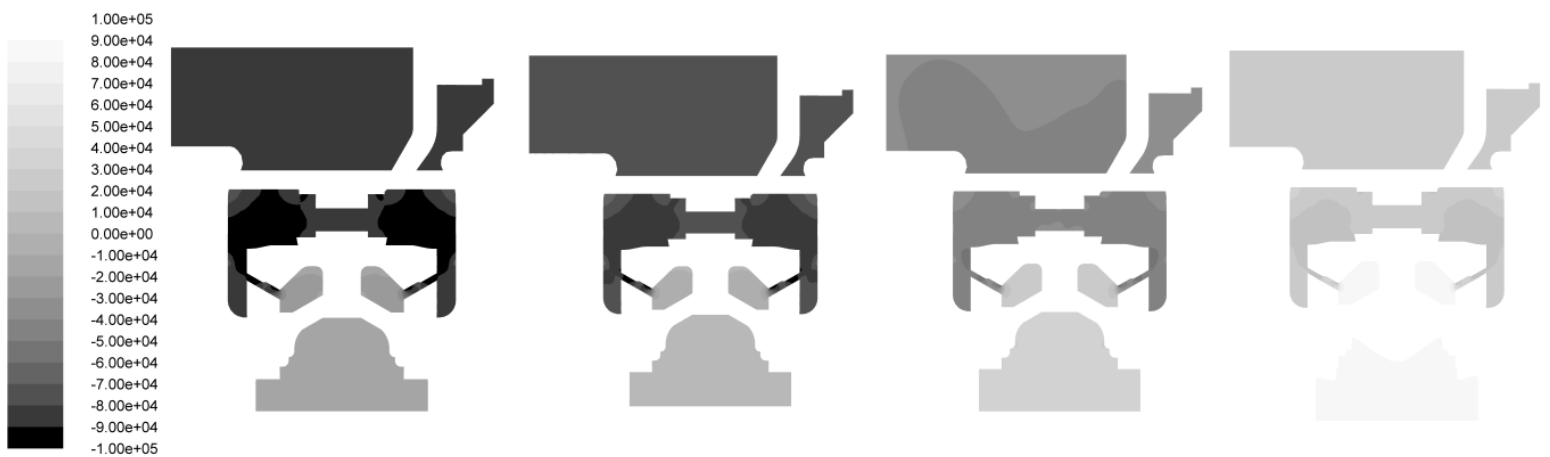

Figure 14. Pressure field $[\mathrm{PaG}]$ in the vicinity of the inlet valve. Case 1 (left) to 4 (right) at $120^{\circ}$ of crank rotation (inlet stroke).

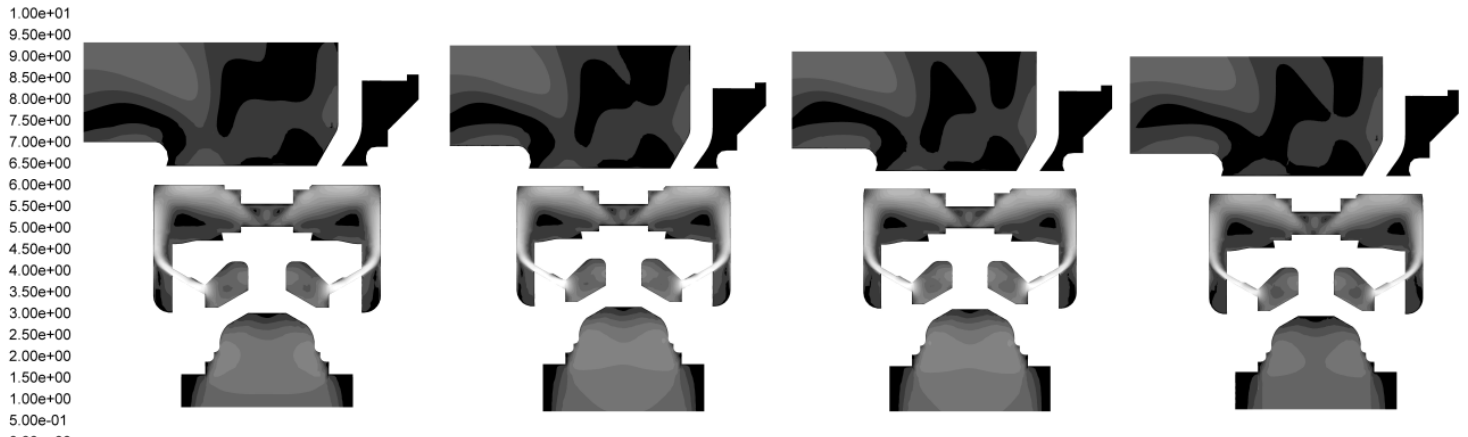

Figure 15. Mixture (water and vapour) velocity field $[\mathrm{m} / \mathrm{s}]$ in the vicinity of the inlet valve. Case 1 (left) to 4 (right) at $120^{\circ}$ of crank rotation (inlet stroke).

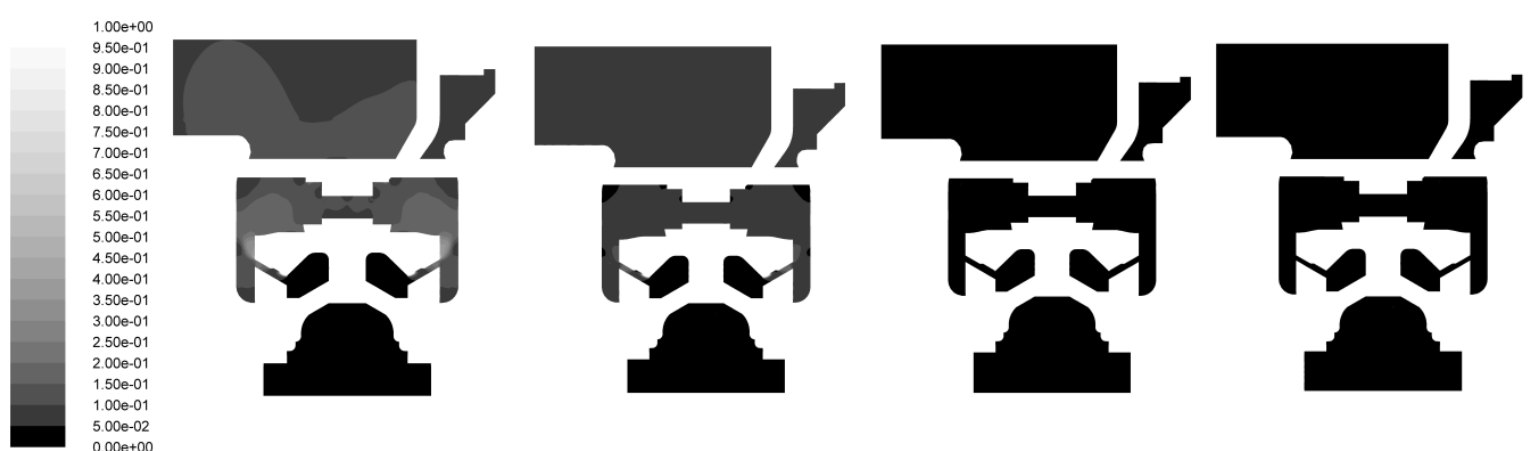

Figure 16. Vapour volume fraction field [-] in the vicinity of the inlet valve. Case 1 (left) to 4 (right) at $120^{\circ}$ of crank rotation (inlet stroke).

\section{Conclusion}

A comprehensive transient CFD model of a PD reciprocating pump was created making use of the Ashok Singhal et Al. [11] cavitation model to simulate the device from incipient to full cavitating conditions. Four cases were studied; they differed only in the inlet pressure boundary condition. The computational model of the PD pump was provided by a UDF which calculated the inlet and outlet valve displacement via integration of the pressure field surrounding the valves.

The model was capable of simulating the phase change in the three conditions of incipient to full cavitation. Good interaction of the phase change with the pressure field and valve lift was also achieved. In case 1, where the higher second phase generation was observed, the vapour trapped in the vicinity of the inlet valve, at the end of the inlet stroke, kept the 


\section{A CFD Study on the mechanisms which cause cavitation in positive displacement reciprocating pumps}

pressure close to the vapour level. The integral of the forces on the valve surfaces prevented the valve from closing until the plunger compressed the vapour and turned it into water again.

Table 3 demonstrates the capability of the model to simulate the behaviour of the non-condensable gas dissolved in the water in terms of the inlet valve opening delay. The model also demonstrated the capability of CFD methodologies in providing the analyst with the information needed to understand the mechanisms leading to cavitation as well as all the information concerning the cavitation dynamics. For instance, this paper showed and discussed the two different types of cavitation affecting PD reciprocating pumps which the numeric model identified; flow induced cavitation and cavitation due to expansion.

It is important to point out to the reader that the work presented in this paper belongs to a bigger project

\section{References}

[1] K. Opitz, E. Schlücker, and O. Schade, "Cavitation in reciprocating positive displacement pumps," in Twentyseventh international pump users symposium, 2011, pp. 27-33.

[2] H. H. Tackett, J. A. Cripe, and G. Dyson, "Positive displacement reciprocating pump fundamentals- power and direct acting types.," in Proceedings of the twentyfourth international pump user symposium, 2008, pp. $45-58$.

[3] R. singh Ragoth and M. Nataraj, "Study on Performance of Plunger Pump at Various Crank Angle Using CFD," IRACST Eng. Sci. Technol. An Int. J. (ESTIJ), ISSN22503498, vol. 2, no. 4, pp. 549-553, 2012.

[4] S. M. Price, D. R. Smith, and J. D. Tison, "The effects of valve dynamics on reciprocating pump," in Proceedings of the twelfth international pump users symposium, 1995, pp. 221-230.

[5] H. Ding, F. C. Visser, and Y. Jiang, "A practical approach to speed up NPSHR prediction of centrifugal pumps using CFD cavitation model," in Proceedings of
The model was accurate enough to quantify the amount of vapour produced and destroyed in the chamber and therefore the efficiency loss throughout the four operational conditions could be calculated.

which is composed of several steps. The first step has been discussed in [10] and dealt with the creation of the single phase model which Has been improved, to the model presented in this paper, by adding the multiphase and cavitation models. The results achieved suggest a further step is required; experimental validation. The authors are already working on a test rig to validate the results shown in this paper. After validation and further improvement the project aims at utilising the CFD tool to optimize the pump design to alleviate cavitation and provide engineers with a reliable and cheap tool to support the design process.

the ASME 2012 Fluids Engineering Summer Meeting., 2012, p. Paper No. FEDSM2012-72282.

[6] J. E. Miller, The reciprocating pump, theory design and use, Second Edi. Krieger publishing company, 1995, pp. $1-467$.

[7] T. Baur, J. Köngeter, and R. Leucker, "Effects of dissolved gas on cavitation inception in free surface flows," in Third International Symposium on Cavitation, 1998, no. April.

[8] K. Opitz and E. Schlücker, "Detection of Cavitation Phenomena in Reciprocating Pumps using a High-Speed Camera," Chem. Eng. Technol., vol. 33, no. 10, pp. 1610-1614, Jul. 2010.

[9] ANSYS, “ANSYS FLUENT User's Guide,” vol. 15317, no. November. ANSYS Inc., 2011.

[10] a. Iannetti, M. T. Stickland, and W. M. Dempster, "A computational fluid dynamics model to evaluate the inlet stroke performance of a positive displacement reciprocating plunger pump," Proc. Inst. Mech. Eng. Part A J. Power Energy, vol. 228, no. 5, pp. 574-584, Apr. 2014.

[11] A. K. Singhal, M. M. Athavale, H. Li, and Y. Jiang, "Mathematical Basis and Validation of the Full 
A CFD Study on the mechanisms which cause cavitation in positive displacement reciprocating pumps

Cavitation Model," J. Fluids Eng., vol. 124, no. 3, p. $617,2002$.

[12] Lord Rayleigh, "VIII. On the pressure developed in a
[13] liquid during the collapse of a spherical cavity," Philos. Mag. Ser. 6, vol. 34, no. 200, pp. 94-98, Aug. 1917.
ANSYS, “ANSYS Fluent Theory Guide,” vol. 15317, no. November. ANSYS Fluent, 2011.

[14] J. P. Franc and J. M. Michel, Fundamental of Cavitation. Kluwer academic publishers, 2004, pp. 1-300. 